\title{
Effect of the Random Error on the Radiation Characteristic of the Reflector Antenna Based on Two-Dimensional Fractal
}

\author{
Na Li, Baoyan Duan, and Fei Zheng \\ Key Laboratory of Electronic Equipment Structure Design of Ministry of Education, School of Mechano-Electronic Engineering, \\ Xidian University, Xi'an, Shannxi 710071, China \\ Correspondence should be addressed to Na Li, lina@mail.xidian.edu.cn
}

Received 13 March 2012; Accepted 17 April 2012

Academic Editor: Dau-Chyrh Chang

Copyright (C) $2012 \mathrm{Na} \mathrm{Li} \mathrm{et} \mathrm{al.} \mathrm{This} \mathrm{is} \mathrm{an} \mathrm{open} \mathrm{access} \mathrm{article} \mathrm{distributed} \mathrm{under} \mathrm{the} \mathrm{Creative} \mathrm{Commons} \mathrm{Attribution} \mathrm{License,} \mathrm{which}$ permits unrestricted use, distribution, and reproduction in any medium, provided the original work is properly cited.

Influence of the random error due to the distortion of the antenna reflector was discussed by using the two-dimensional fractal model. Firstly, a mathematical model of the random error was presented to investigate its impact on the radiation characteristic of the antenna, which was filtered from the two-dimensional fractal by using the wavelet analysis method. Based on the error model, a density formulation of the far field of the antenna with random error was deduced. Finally, a $3.7 \mathrm{~m}$ and a $3 \mathrm{~m}$ antennas were used to validate the proposed models, and a novel simulation method was put forward to remedy the deficiencies of existing software tools for the antenna with error. Results of the proposed methods showed a good agreement with the experimental data, and the existence of error broadened the beam width and raised the sidelobe level of the antenna, while it had little influence to the antenna gain. By comparison on results of the different scales error, the main influencing parameter of error was found out, which can provide the theoretical foundation for the control of the antenna surface working.

\section{Introduction}

Reflector antennas are of great important in microwave communication and relay system engineering. However, the reflector surface of the antenna in engineering application will never be fully consistent with the ideal surface, and there is necessarily some error. The error factors (random error and system error), as the significant factors affecting the antenna radiation characteristic, are the neck bottle against its rapid development [1-3]. Since Ruze published the first work on this subject and pursued the effect of small phase and amplitude error on the average sidelobe level [1]. Influence of the error on the radiation characteristic of reflector antenna has been studied by a number of authors and achieved significant achievements [4-7]. But researches on effect of the random error on the radiation characterize were mainly focused on the system error, while the random error in micro-nanoscale was paid little attention.

It is generally assumed that the random error is evenly distributed on the reflector surface. So some random functions were chosen to simulate the error. But the random error of engineering reflector surface is neither totally random nor deterministic, and the altitude distribution, slope, and curve of the error dependence on the resolution and the sampling length of the measuring instrument, which results in the description value, is not unique for the same rough surface $[8]$. With the advent of fractal geometry $[9,10]$, a new tool has become available for describing random error caused by the antenna manufacturing process. In our previous work, a one-dimensional fractal was used to analyze the effect of rough surface on the waveguide [11]. However, the scope of its applicability is limited, because its math function has a definite form. In this paper, a two-dimensional fractal is used to simulate the rough surface, which requires no specific forms and has more expansibility and wider application scope than one-dimension fractal.

Large caliber and high precision characteristic of the reflector antenna result in the high productive cost and long productive cycle. So bulk production is a romantic method for it, and the verification of related theory mainly uses modeling and simulation methods. But any existing commerce software can analyze the nonideal antenna, which constraints 
the verification and development of the related theory [1214]. Aiming at these problems, a new simulation method of the reflector antenna was represented in this paper. The random error was considered in the process of the antenna modeling and analysis process by adding the interrelated software module compiled into existing commerce software. The value of random error was determined based on the machining technology and the working environment of the reflector antenna. It expanded the applicability scope of the commerce software and improved the analysis precision of antenna.

For research on the effect of the error, most existing relative literatures revised the radiation characteristic formulation after the antenna was manufactured and tested, but not in the process of the antenna design even before [15-19]. The existing experimental methods, which can gain engineering experience, still did not find the influence of the random error essential to the antenna performance, to say nothing of building the quantitative influence relations between them [20]. What is worse, is that the reference data for the revision was obtained by assuming some probability models, which inevitably resulted in inaccurate analytical results.

In this paper, the effect of the random error on the reflector antenna was found to increase the phase error. A formulation of density of the far field based on the random error model was deduced, in which the error was determined according to the machining technology and the working environment of the reflector antenna.

\section{Effect of the Random Error on the Radiation Characteristic}

2.1. Random Error Modeling. It is generally though that the error on the reflector surface of the antenna is random because of its uncertainty in production and distribution. So the random error was simulated by random functions in the existing research [1-10]. However, the measure parameters of the random model depended on the resolution of the measuring instrument, which made the measure value not unique for an identified surface. In order to resolve the contradiction with the finiteness of the measuring instrument resolution and the correlation of characterizng parameters, the fractal function was used [20-23]. A mathematic model of the random error based on the two dimensional fractal was built as follows:

$$
\begin{aligned}
z(x, y) & \\
= & L\left(\frac{G}{L}\right)^{(D-2)}\left(\frac{\ln b}{M}\right)^{1 / 2} \sum_{m=1}^{M} \sum_{n=0}^{n_{\max }} b^{(D-3) n}\left\{\cos \phi_{m, n}\right\} \\
& -\cos \left[\frac{2 \pi b^{n}\left(x^{2}+y^{2}\right)^{1 / 2}}{L} \cos \left(\tan ^{-1}\left(\frac{y}{x}\right)-\frac{\pi m}{M}\right)+\phi_{m, n}\right],
\end{aligned}
$$

where $x$ is the abscissa and $y$ is the ordinate of the error surface. $L$ is the sampling length of the error. $G$ is the frequency independent scaling parameter and $D$ is the fractal dimension. $b$ is the fundamental spatial frequency. $N$ and $M$ are the numbers of tones, and $\phi_{m, n}$ is a phase term that has a uniform distribution over the interval $[-\pi, \pi]$. The purpose of normalization is giving the model function of the error with an arbitrary $R_{a}$, which is the root mean square (RMS) of this surface error. When $D=2.5$ and $b=1.6$, the value of $n$ drops from 2048 to 1024 , which is equivalent to that the resolution drops by one half, while the surface contour of the error is still similar to its former state. It shows that the dimension of the error function was a parameter not concerning the resolution of the measuring instrument and the model can be used to represent the essential of the surface error.

In the present paper, the random error was simply modeled by two-dimensional fractal grooves of one unit length by MATLAB 7.1, and two parameters were introduced to describe the error. As illustrated in the Figures 1 and 2, one was the parameter $D$, which was the fractal dimension; the other was the parameter $b$, which was the fundamental frequency in space and determined the surface density. Figure 1 showed two sample improved W-M surfaces. In Figure 1(a), $b=1.1$ was used, which resulted in successively added cosine shapes whose period and amplitudes were moderately spaced apart. When $b=1.6$, the difference between the periods and heights of consecutive cosine shapes was greater. This coarse separation of roughness scales led to the easily discernable scales of bumpiness seen in Figure 1(b). Next, several improved W-M surfaces were created with parameter $D$ varying from 2.5 to 2.8 as shown in Figure 2 . Different from the parameter $b$, the parameter $D$ affected the dimension of the surface, and the increasing of the parameter $D$ caused a remarkable expansion in the detailed information of the surface roughness.

Based on the microwave surface theory, $98.2 \%$ of the current concentrates in the scope of the $4 \pi$ from the surface. Therefore, the current flowing in the depth of the $4 \tau$ from the surface rather than the exact shape of the surface profile is considered as a more reasonable view. The error on the scale whether too large or too small has little influence on the electrical properties. Depending on the skin depth theory, the error affecting the spheres was determined based on the antenna working frequency, and the affecting sphere was regarded as an assessment reference surface [24]. The random error model was separated from the reference surface by the filtering process as follows:

$$
\begin{gathered}
z_{1}(x, y)=z(x, y) \cdot h(x, y), \\
Z_{1}\left(\omega_{x}, \omega_{y}\right)=Z\left(\omega_{x}, \omega_{y}\right) H\left(\omega_{x}, \omega_{y}\right), \\
H\left(\omega_{x}, \omega_{y}\right) \\
= \begin{cases}1 & \omega_{\text {inf } x} \leq\left|\omega_{x}\right| \leq \omega_{\text {sup } x}, \omega_{\text {inf } y} \leq\left|\omega_{y}\right| \leq \omega_{\text {sup } y}, \\
0 & \omega_{x}<\omega_{\text {inf } x} \cup \omega_{x}>\omega_{\text {sup } x}, \omega_{y}<\omega_{\text {inf } y} \cup \omega_{y}>\omega_{\text {sup } y},\end{cases}
\end{gathered}
$$

where $z_{1}(x, y)$ is the affecting error of the antenna, $z(x, y)$ is the original mathematic model of the random error, and 


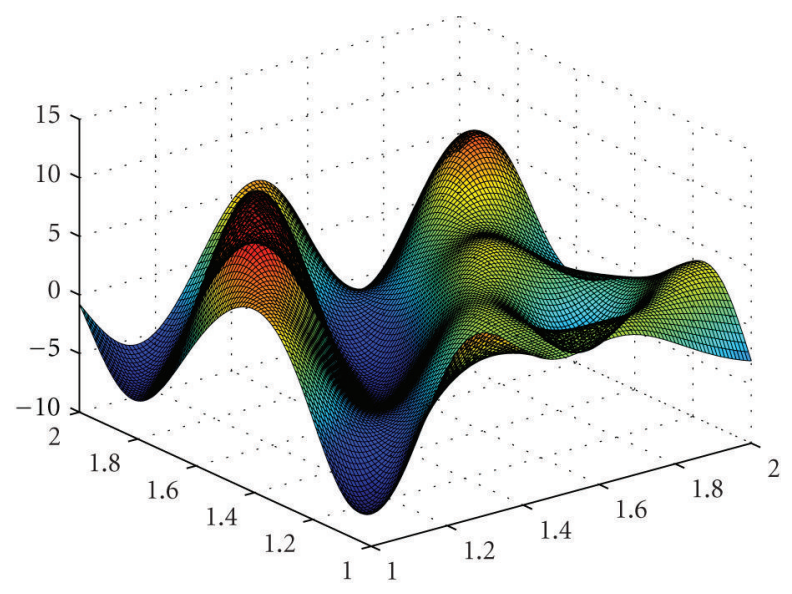

(a) $D=2.1, b=1.1$

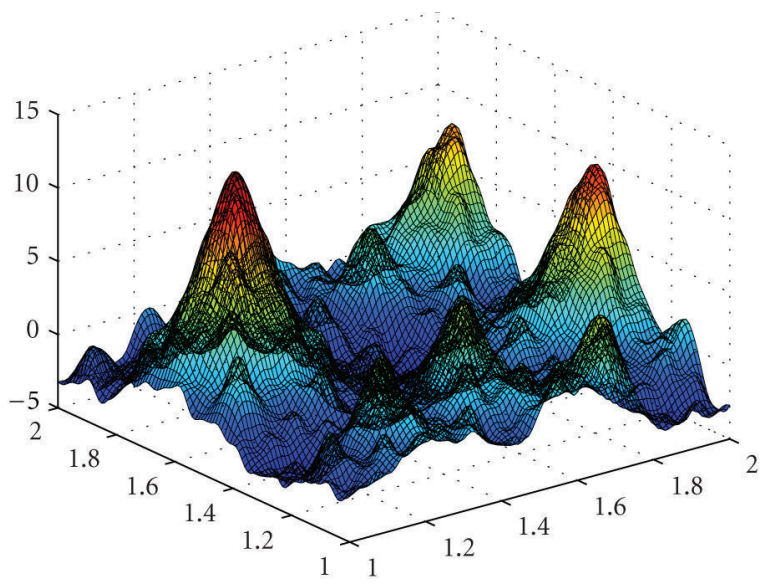

(b) $D=2.1, b=1.6$

Figure 1: Two W-M surfaces produced using different rough surface densities.

$h(x, y)$ is the transfer function of the filter, and $Z_{1}\left(\omega_{x}, \omega_{y}\right)$, $Z\left(\omega_{x}, \omega_{y}\right)$, and $H\left(\omega_{x}, \omega_{y}\right)$ is the Fourier transforms of them. $\omega_{\text {inf } x}$ and $\omega_{\text {sup } x}$ are the upper and lower frequency limit of the cutoff frequency, which is determined by the working frequency of the antenna.

2.2. Radiation Characteristic of the Reflector Antenna with Error. As shown in Figure 3, the antenna has a diameter of $D=2 a$, and the focal length of $f$. Because the reflector surface locates in the far-field region of the antenna feed, the electromagnetic wave is emitted by the feed propagated through the reflector surface and sent to the aperture surface. $\Delta z$ denotes the random error on the reflector surface. Because of the finite size of the $\Delta z$, its influence on the magnitude of electromagnetic wave on the aperture surface can be neglected. So the viewpoint that the existence of random error only changes the axial displacement on the aperture surface is feasible. It makes the phase difference turns into the function of the location. So the aperture surface of antenna will no longer be an equiphase surface.

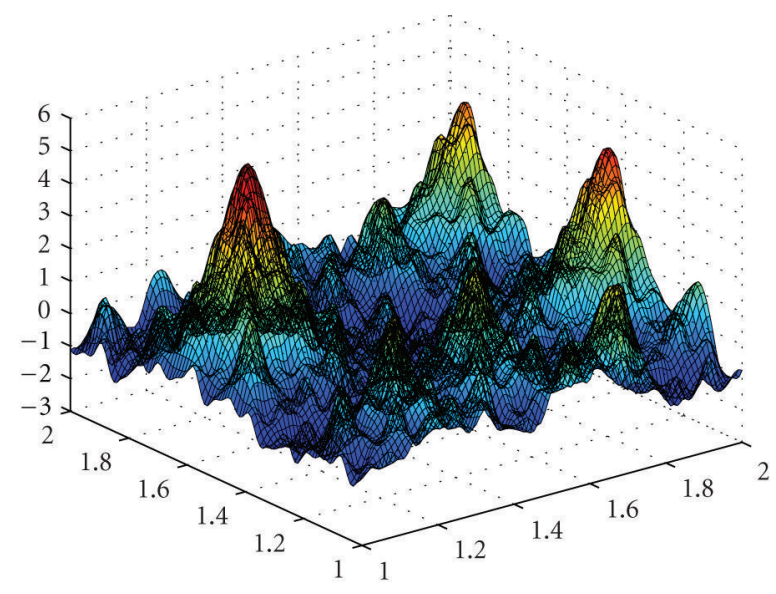

(a) $D=2.5, b=1.6$

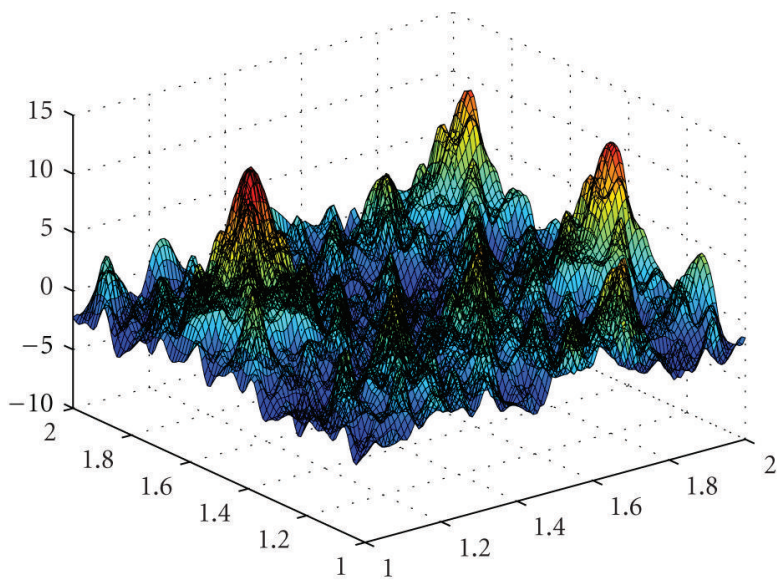

(b) $D=2.8, b=1.6$

Figure 2: Two W-M surfaces produced using different fractal dimensions.

And the radiation pattern of the antenna will change too [22].

$$
E(\theta, \phi)=\iint_{S^{\prime}} f\left(\xi, \phi^{\prime}\right) e^{j \Delta \varphi} e^{j \varphi\left(\rho^{\prime}, \phi^{\prime}\right)} \rho^{\prime} d \rho^{\prime} d \phi^{\prime},
$$

where $E(\theta, \phi)$ is the density of far field in $p$ point, and $f\left(\xi, \phi^{\prime}\right)$ is the distribution function of the antenna aperture on the caliber surface $S^{\prime} . e^{j \Delta \varphi}$ denotes the phase error caused by the random error on the reflector surface, while $e^{j \varphi\left(\rho^{\prime}, \phi^{\prime}\right)}$ is the phase error caused by the positional and direction error of the antenna feed. The equation for the phase error can be expressed as follows:

$$
\Delta \varphi=4 \pi z_{1}(x, y) \cos ^{2} \frac{(\xi / 2)}{\lambda},
$$

where $z_{1}(x, y)$ is the random error model on the reflector surface of the antenna, and $\lambda$ is the working length of it. The error function in (2) is used as the axial displacement and substituted in (3), and (4) is used to combine the error and the radiation characteristic of the antenna to obtain the gain degradation coefficient of the antenna with respect to the distorted reflector. 


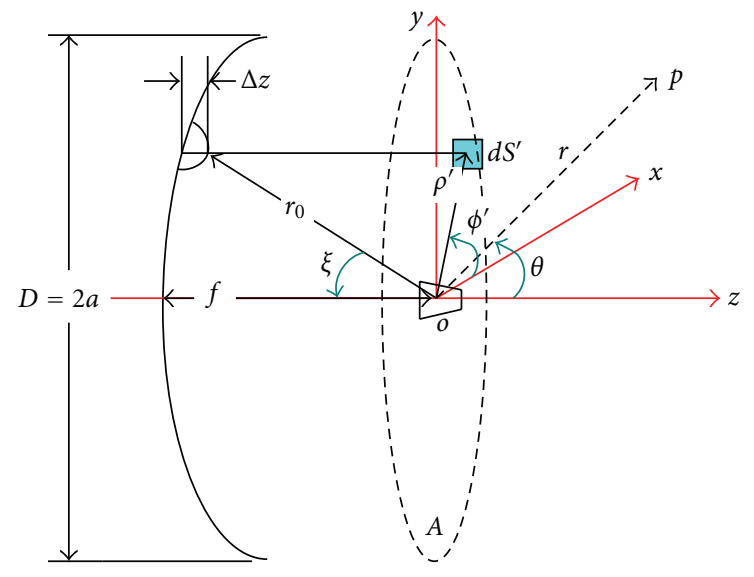

FIGURE 3: Schematic diagram of the reflector antenna with error.

\subsection{Simulation of the Reflector Antenna with Random Error.} The large caliber and high precision characteristic of the reflector antenna result in high costs and long cycle in antenna manufacturing, and mass production of antenna for experiments is difficult or impractical. So that, the simulation research attracts the interest of scientists in this field because of its good repeatability and low cost. But any existing commerce software can analyze the nonideal antenna, and the structural and electromagnetic analyze is carried out separately, which will cause the mismatch in structural and electromagnetic mesh and reduce the final analysis accuracy.

The key to these problems is how to add the error into the process of the antenna simulation and integrates the structural and electromagnetic analysis. A flowchart of the novel simulation method was given as shown in Figure 4. Firstly, the machining error is added into antenna model in the process of geometric modeling, and the error was set by the description precision. And then, when the structure analysis of the antenna is carried out, the loading error was added, and the deformed structure model of the antenna is gained by the structure analysis. On the other hand, the assemble error is added into the deformed model with the model modifying module. Finally, the deformed structure model is translated into the electromagnetic analysis model, and the electrical property of the antenna is obtained by the electrical performance analysis.

The new simulation method is proposed to verify the correctness and reliability of the modeling and analysis method mentioned above. The error models were built to refer to the engineering data, which ensures the accuracy of the model. If the electrical properties result met the design requirement, the cycle is completed. Otherwise, the threetypes error will be remedied to gain the ideal result.

\section{Testing of the Theory}

In order to validate the presented modeling and simulation methods, the applications in the $3.7 \mathrm{~m}$ and $3 \mathrm{~m}$ cassegrain reflector antennas of frequencies in the $\mathrm{Ku}$ band were

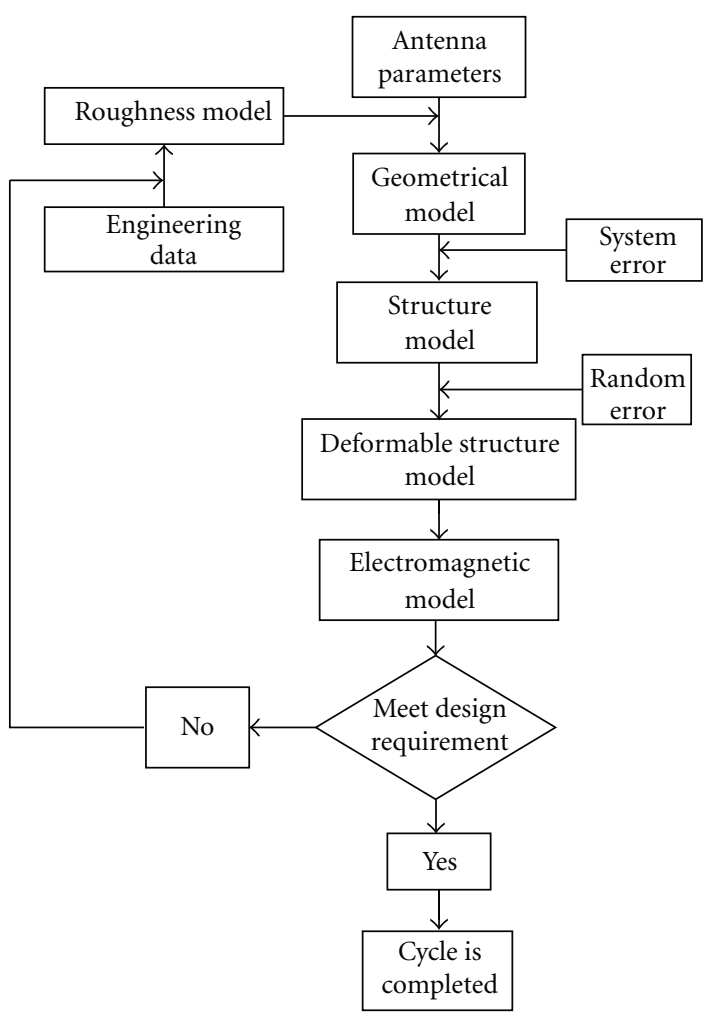

Figure 4: Flow chart of the novel simulation method.

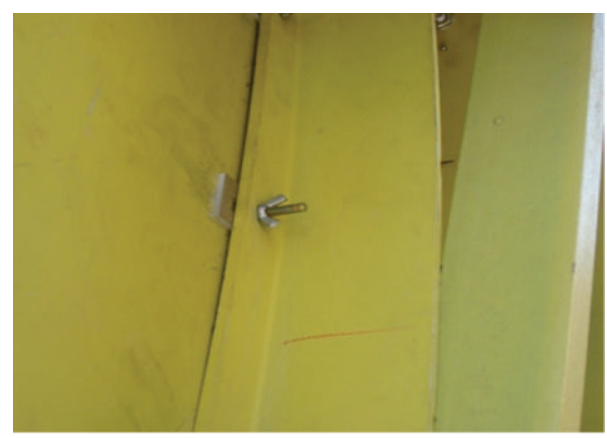

Figure 5: Partial view of imposing shim on the antenna back.

demonstrated. In one hand, an artificial deformation on the antenna surface was accomplished by imposing some shims on the antenna backs as shown in Figure 5, and point 1 to point 13 were the positions of the shims. Based on the measuring data of the deformable surface, the random error model was built, and the electric properties of the antenna can be calculated by (3). On the other hand, the electric properties of the antenna also can be obtained by the simulation method shown in Figure 4. The accuracy and superiority of the proposed methods were checked by comparing the calculation and the simulation results to the test data of the antenna with deformable surface.

Firstly, a comparison of test data and result of the proposed method of the $3.7 \mathrm{~m}$ antenna was made. The caliber of the main reflector surface and the auxiliary reflector surface of the antenna are $3.7 \mathrm{~m}$ and $0.44 \mathrm{~m}$, respectively. 


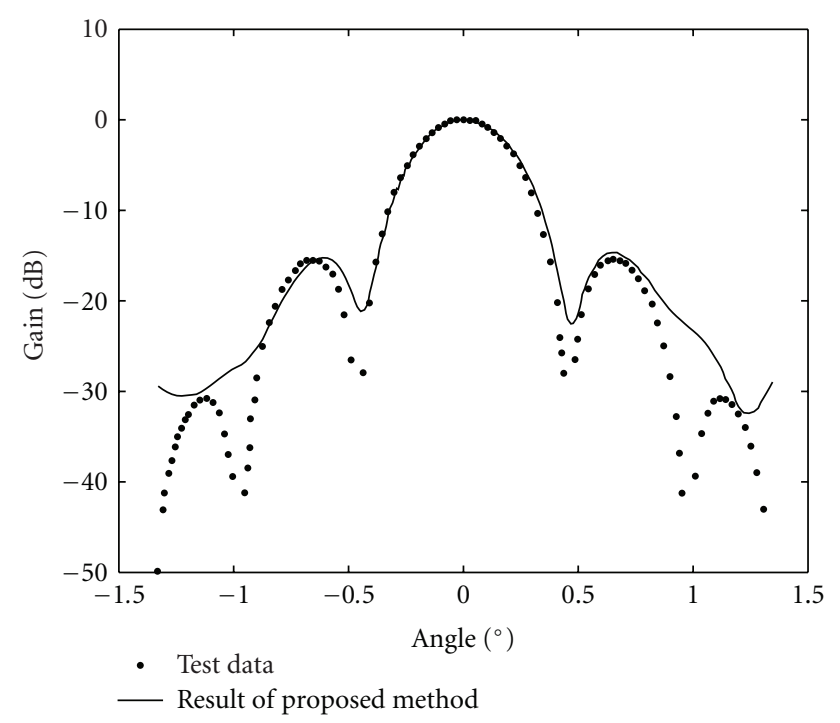

Figure 6: Comparison of the test data and results of the proposed method.

In the experiment, the antenna was used as the receiving one and its working frequency was $12.5 \mathrm{GHz}$. As shown in Figure 6, the gain and the first sidelobe of the antenna along the angle were all highly consistent with the test data in the area of the main lobe, which manifest the accuracy of the proposed methods. The other sidelobes differed obviously in the phase and amplitude quantification because the theory was based on the antenna working in the ideal circumstance, while such ideal experiment conditions were not readily reproduced in practical reflector antenna test.

For the large caliber antenna, the calculation efficiency is an important question, which concerns whether the calculation can be completed or not. Table 1 showed a comparison of CPU times among four mathematical models for thez random error of the reflector antenna. As expected, the time for the Gaussian and exponential functions were basically similar, and the fractal is about 10-times slower than Gaussian and exponential function due to its abundant details information. Although fractal function was more efficient if our interest is limited in the computation result, the random error (RE) model remains useful in the study of the electrical properties, irregular structures modeling, and iterative solvers. When compared with the two Monte Carlobased peers, fractal took about 4-times more CPU time than $\mathrm{RE}$ for one surface realization, and about 10-times more than Gaussian and exponential functions. However, with a view to the large number of runs for Monte Carlo simulation to achieve a satisfactory convergence (e.g., fractal takes 432000 s and RE takes 95000s for 2000 runs), the RE model was actually much faster than the original fractal function.

In order to analyze the different influence of the different amplitude of random error on the radiation characteristic of the reflector antenna, and find out the predominant influence in the causation of the specific performance of the antenna, a comparison of the different amplitude of the random error was made for the $3 \mathrm{~m}$ reflector antenna. As shown

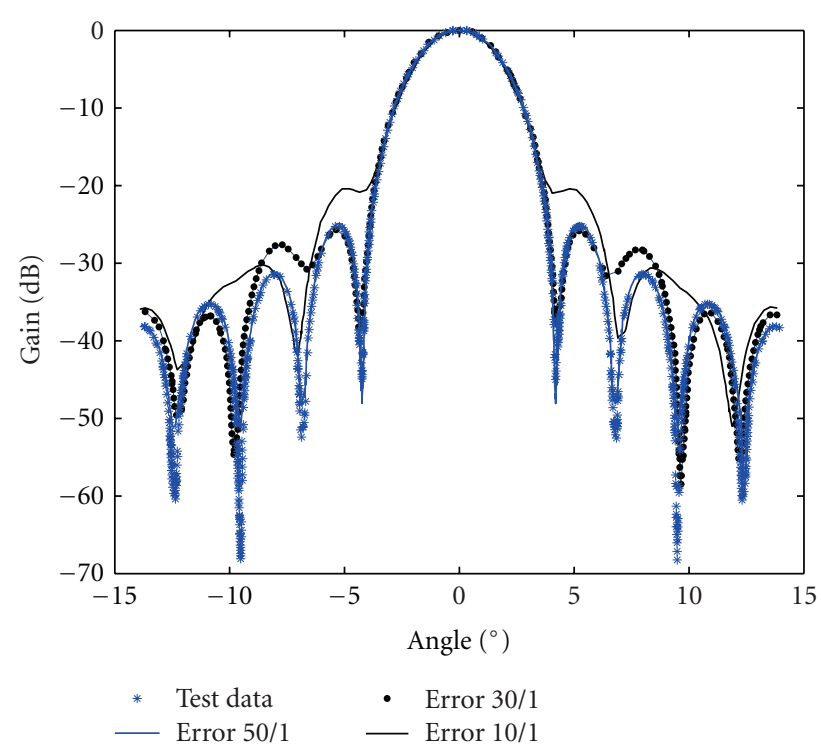

FIGURE 7: Comparison of the different amplitude error to the test data.

TABle 1: CPU time comparison for different mathematical models of the random error.

\begin{tabular}{lcccc}
\hline Math function & Gaussian & Exponential & Fractal & RE \\
\hline CPU time (one run) & $23.8 \mathrm{~s}$ & $18.7 \mathrm{~s}$ & $216 \mathrm{~s}$ & $47.5 \mathrm{~s}$ \\
$(1200$ runs) & $47600 \mathrm{~s}$ & $37400 \mathrm{~s}$ & $432000 \mathrm{~s}$ & $95000 \mathrm{~s}$ \\
\hline
\end{tabular}

in Figure 7, increasing of the error amplitude broadened the beam width and raised the sidelobe level of the antenna, while it had little impact on the antenna gain. And when the amplitude of the roughness approximates the tenth part of the working wavelength, the antenna has the most influential effect. The finding suggested that determining the strongest factor based on the operation frequency and reducing it in the process of the manufacture was an effective way to make sure of the effectiveness of antenna design, which will reduce the production cost and shorten the circle for antenna production as well.

\section{Conclusions}

In this paper, a mathematic model of the random error was built to study effect of the random error on the radiation characteristic of the reflector antenna. The superiority in calculation accuracy and efficiency of the proposed methods were proved by the comparison of the random error model to the test data with the examples of the $3 \mathrm{~m}$ and $3.7 \mathrm{~m}$ reflector antennas. For the same reflector antenna, the gain reduction coefficient obtained by proposed methods is higher than that gained by current methods, due to the fact that the proposed methods inherently took the random error and systemic error into consideration simultaneously. In addition, the influence degrees of different amplitudes of the random error were discussed, which was of great theoretical and practical significance in the reflector antenna designing and 
manufacturing, which has not been fully discussed in the past. The importance of the proposed methods in the paper was directly expressing the electromagnetic performance in terms of the mechanical parameters, which can be taken as the reference for the general research on the other mechanical factors.

Besides, two problems are still unsettled. Firstly, as one of the parameters, which can fully describe the morphology of the random error, the parameters of the random error model should have some inter-relationships with the traditional judge system to manifest the continuity of the science. On the other hand, it is our experience used in the comparisons, qualitative methods tend to be faster, less expensive, and more likely to provide useful answers to important questions that lead to superior design. We deduced the formulation of density of the far field based on the error model. But the qualitative analysis of comparison results is not quite finished. Further study is needed to give a qualitative comparison to meet the logic, preciseness, accuracy, and application of this science.

\section{Acknowledgments}

This work is supported by the Fundamental Research Funds for the Central Universities (no: JY10000904019), the Key Projects (no: 51035006), and the National Natural Science Foundation of China (no: 50975217). Particular thanks are also due to Dr. L.W. Song and P. Li who have provided technical aid and generous support.

\section{References}

[1] X. J. Wang, "On the design of a reflector antenna," Inverse Problems, vol. 12, pp. 351-357, 1996.

[2] R. Bracewell, "Tolerance theory of large antennas," IEEE Transactions on Antennas and Propagation, vol. 9, pp. 49-58, 1961.

[3] B. Y. Duan and C. S. Wang, "Reflector antenna distortion analysis using MEFCM," IEEE Transactions on Antennas and Propagation, vol. 57, no. 10, pp. 3409-3413, 2009.

[4] C. S. Wang, B. Y. Duan, and Y. Y. Qiu, "On distorted surface analysis and multidisciplinary structural optimization of large reflector antennas," Structural and Multidisciplinary Optimization, vol. 33, no. 6, pp. 519-528, 2007.

[5] S. Sinton and Y. Rahmat-Samii, "Random surface error effects on offset cylindrical reflector antennas," IEEE Transactions on Antennas and Propagation, vol. 51, no. 6, pp. 1331-1337, 2003.

[6] J. Ruze, "Antenna tolerance theory-a review," Proceedings of the IEEE, vol. 54, pp. 633-640, 1966.

[7] B. Y. Duan, Y. H. Qi, G. H. Xu, and W. T. Wang, "Study on optimization of mechanical and electronic synthesis for the antenna structural system," Mechatronics, vol. 4, no. 6, pp. 553-564, 1994.

[8] M. V. Lukić and D. S. Filipovic, "Modeling of 3-D surface roughness effects with application to $\mu$-coaxial lines," IEEE Transactions on Microwave Theory and Techniques, vol. 55, no. 3, pp. 518-525, 2007.

[9] B. B. Mandelbrot, The Fractal Geometry Of Nature, Freeman, San Francisco, Calif, USA, 1983.

[10] N. Li and F. Zheng, "Effect of micro/nano-scale rough surface on power dissipation of the waveguide:model and simulate,"
Journal Of Nanoscience and Nanotechnology, vol. 11, pp. 1-5, 2011.

[11] J. Ruze, "The effect of aperture errors on the antenna radiation pattern,” Il Nuovo Cimento, vol. 9, no. 3, pp. 364-380, 1952.

[12] J. W. Kim and B. S. Kim, "Computation of the average power pattern of a reflector antenna with random surface errors and misalignment errors," IEEE Transactions on Antennas and Propagation, vol. 44, no. 7, pp. 996-999, 1996.

[13] K. Bahadori and Y. Rahmat-Samii, "Characterization of effects of periodic and aperiodic surface distortions on membrane reflector antennas," IEEE Transactions on Antennas and Propagation, vol. 53, no. 9, pp. 2782-2791, 2005.

[14] C. A. Gueriny, M. Holschneider, and M. Saillardz, "Electromagnetic scattering from multi-scale rough surfaces," Waves in Random Media, vol. 7, pp. 331-349, 1997.

[15] X. Chen, J. Raja, and S. Simanapalli, "Multi-scale analysis of engineering surfaces," International Journal of Machine Tools and Manufacture, vol. 35, no. 2, pp. 231-238, 1995.

[16] G. Beylkin, R. Coifman, and V. Rokhlin, "Fast wavelet transforms and numerical algorithms," Communications on Pure and Applied Mathematics, vol. 44, pp. 141-183, 1991.

[17] W. J. Jasper, S. J. Gamier, and H. Potlapalli, "Texture characterization and defect detection using adaptive wavelets," Optical Engineering, vol. 35, no. 11, pp. 3140-3149, 1996.

[18] M. S. Zarghamee, "On antenna tolerance theory," IEEE Transactions on Antennas and Propagation, vol. 15, pp. 777781, 1967.

[19] T. B. Vu, "Influence of correlation interval and illumination taper in antenna tolerance theory," Proceedings of the IEEE, vol. 116, pp. 195-202, 1969.

[20] J. H. Yao, Y. D. Zhang, J. M. Xiao, and D. B. Ge, "Comparison Of EM scattering from both statistic and fractal models of random Rough sea surface," Journal of Microwares, vol. 16, pp. 144-148, 1990.

[21] A. Majumdar and C. L. Tien, "Fractal characterization and simulation of rough surfaces," Wear, vol. 136, no. 2, pp. 313$327,1990$.

[22] M. Ausloos and D. H. Berman, "Multivariate weierstrassmandelbort function," Proceedings of The Royal Society of London A, vol. 400, no. 1819, pp. 331-350, 1985.

[23] B. Josso, D. R. Burton, and M. J. Lalor, "Frequency normalized wavelet transform for surface roughness analysis and characterization,” Wear, vol. 252, pp. 491-500, 2002.

[24] Q. Chen, S. Yang, and Z. Li, "Surface roughness evaluation by using wavelets analysis," Precision Engineering, vol. 23, no. 3, pp. 209-212, 1999. 

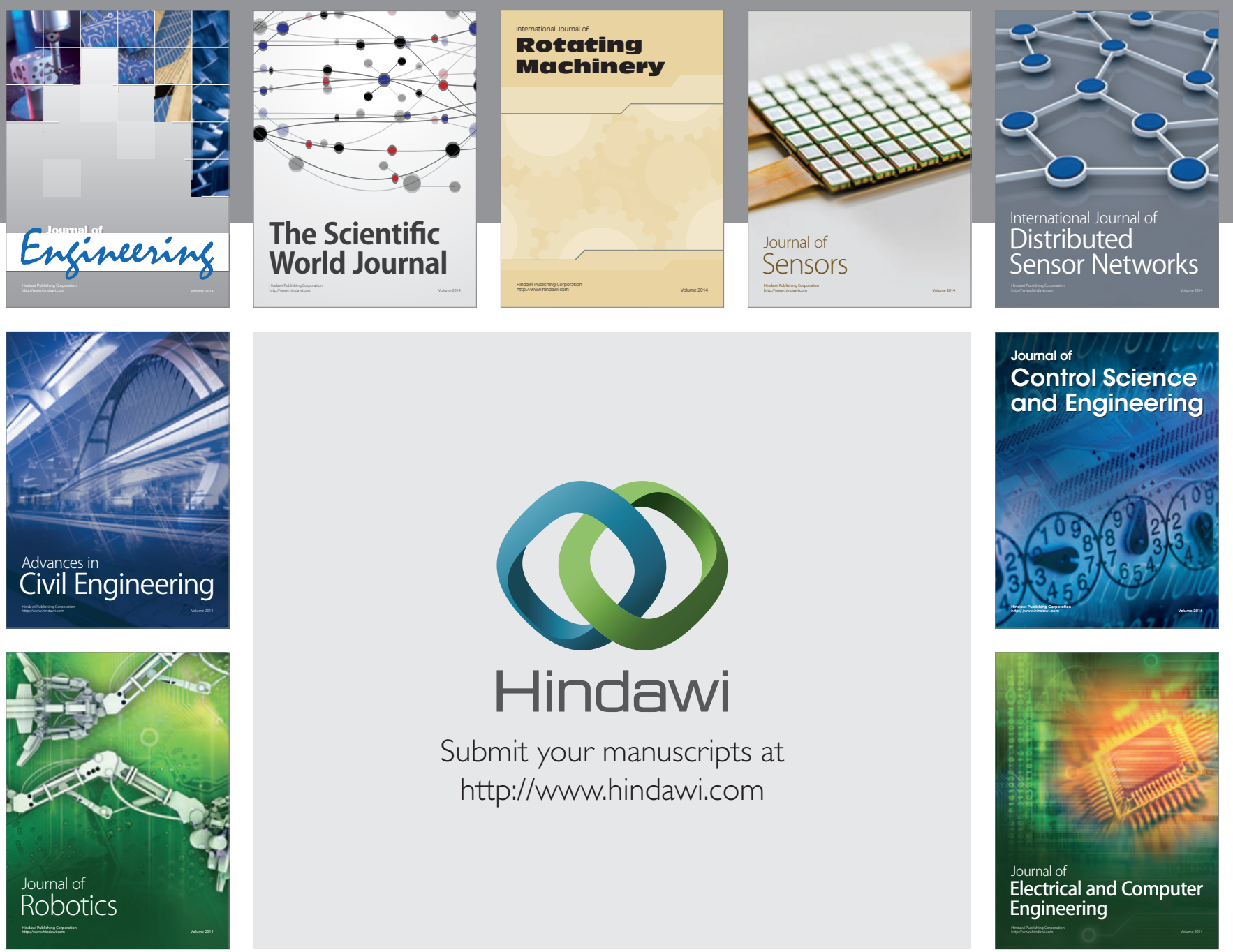

Submit your manuscripts at

http://www.hindawi.com
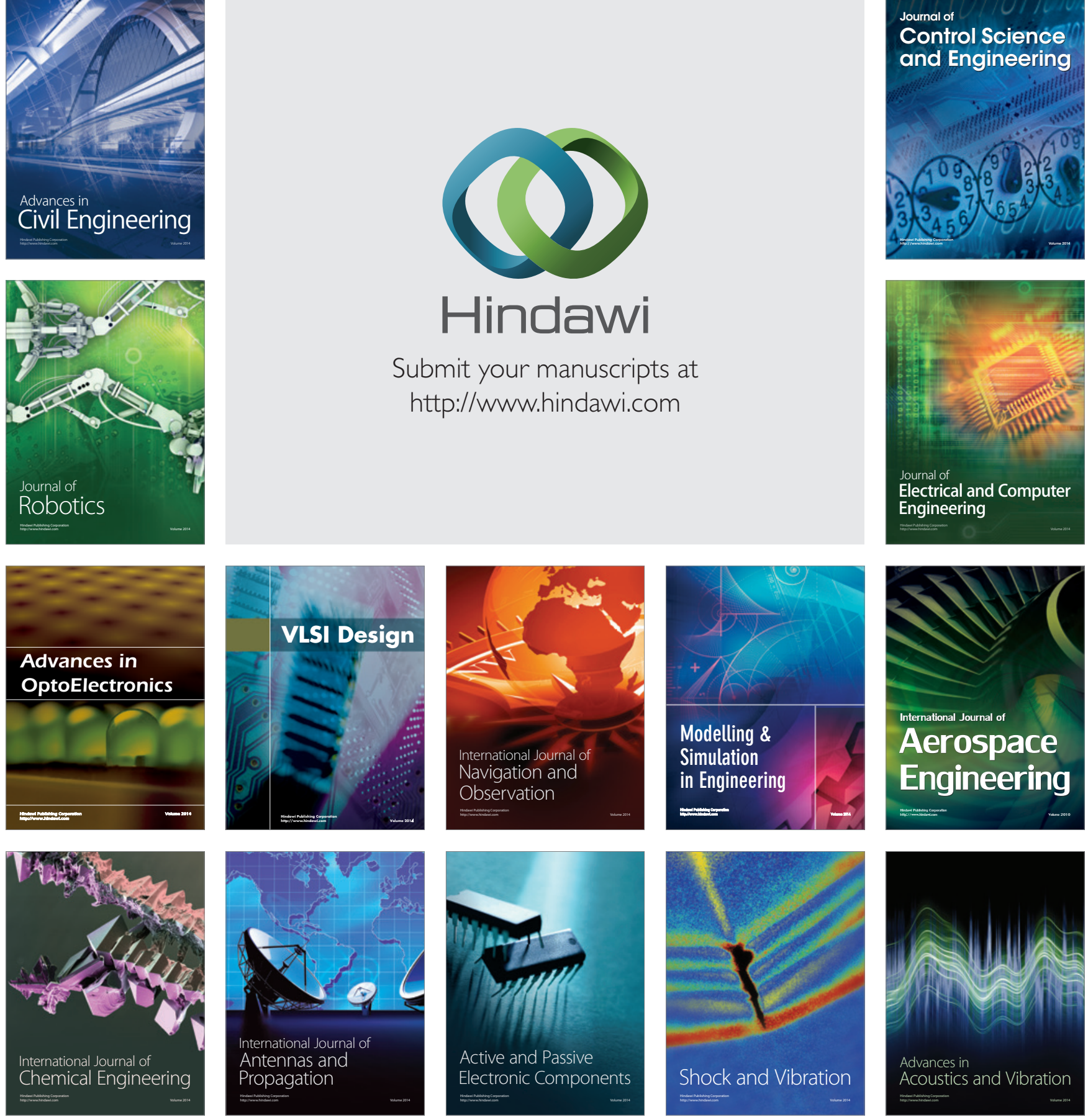